\title{
Demand oriented Modelling of coupled Energy Grids
}

\author{
Jörn Benthin ${ }^{1}$ Annika Heyer ${ }^{1}$ Philipp Huismann ${ }^{1}$ Michael Djukow ${ }^{1}$ Anne Hagemeier ${ }^{2}$ Klaus Görner ${ }^{1}$ \\ ${ }^{1}$ Gas- und Wärme-Institut Essen e.V., Germany, \\ \{benthin, heyer, huismann, k.goerner\}@gwi-essen.de \\ ${ }^{2}$ Fraunhofer UMSICHT, Germany, anne.hagemeier@umsicht. fraunhofer. de
}

\begin{abstract}
This paper describes the development of a modular approach for modelling and simulation of coupled energy grids within different kinds of settlement structures. One presented thesis is that the spatial distribution of the demand structure is given by the urban framework and should be the origin of the modelling of coupled energy grids on the distribution level. Thus, the logic is that the grid is developing towards the given and developing demand structure and not vice versa. The defined spatial distribution delivers the loss relevant lengths between the consumers and producers, which are needed for pipes and cables.

Following these assumptions, a modular approach was realised by creating a so-called GridConstructor. This constructor allows it to easily build user defined urban frameworks and combine them with a single grid or multiple grids (electricity, gas, heat). These grids can be coupled via different systems. In conclusion, first results of coupled grid simulations are presented.
\end{abstract}

\section{Keywords:}

Thermodynamic and energy systems applications, Large-scale system modelling

\section{Introduction}

The ongoing integration of renewable energy sources into the different energy grids is one of the major tasks for the next decades. The overall goal behind this integration is the decarbonisation of the energy consumption in the different structural sectors (industrial, service, residential, mobility). Due to the highly volatile and increasing power input of the renewable energy sources, the need for coupled energy grids and flexibilities is rising (Behnert, 2018).

Especially on the distribution level, the question arises how the different grid designs (electricity, gas, heat) will look like and how these networks are going to connect and interact. To find the ecological and technological optimum, different coupled design options have to be analysed.

To address these questions, different libraries and software tools are available. In general, these tools share one common approach. This approach is defined by the modelling hierarchy, which sets the grid structure as a fixed boundary and not the urban framework.

The natural process in the development of cities and districts is that the demand structure is given by the urban framework and its developing demand and decentralised production. The energy grids have to develop towards these needs and therefore the grid design, connections and interactions are the variables of this adjustment process. The given or designed spatial structure of the settlement delivers the loss relevant lengths between the consumers, producers and the demand density, which are needed for the sizing of pipes, cables and the design of the resulting networks.

These assumptions were used in the research project IntegraNet (IntegraNet, 2018) to develop a modular approach for modelling urban frameworks at the distribution grid level.

The TransiEnt (TransiEnt, 2018) library developed within the project Transient.EE (Andresen, 2017) by the Hamburg University of Technology was used for the work presented in this paper.

\section{GridConstructor}

\subsection{Modelling Philosophy}

The future energy grid will undergo considerable changes through decentralisation, an increasing share of renewable energy supply and sector coupling technologies. In order to investigate the potentials of these technologies and other feasible innovations in grid design, the modelling of energy grids needs to be able to map the existing structure as well as future options. Thus, a modelling philosophy for energy grids should be based upon flexibility to allow the research of multiple configurations of technologies and types of energy supply.

In case of energy grids, the network structure, as in routing and connections of pipes and cables to consumers, is not fixed and can change depending on the energy supply strategy and time. For example, it might happen that an energy concept for a district based entirely on decentralised oil heating systems is converted to an energy supply via district heating, $\mathrm{PtH}$ 
or gas heating. In this case the overall grid structures are changed and extended, but the demand structure, as in the distances between consumers and their location, stays constant. Based upon this, the modelling of energy grids only from a standpoint of grid structure with fixed types of pipes and cables, that are derived from the given technologies, is no optimal solution to target research projects regarding the development of future energy grids. The use of such models would make simulations with changing technologies difficult since changes in energy generation can involve a change in grid structure. Instead, modelling based on demand structure is more feasible, since it will usually not undergo modifications with changed technologies or grid structure.

\subsection{Structure}

Based on a demand structure oriented modelling, the GridConstructor (GC) has been conceptualised for Modelica. The GC uses reoccurring patterns in the demand structure to model the energy grid in a flexible and user-friendly way. Based upon settlement types defined in (Blesl, 2001), energy grids can be described using several grid segments with changing numbers of consumers. These grid segments contain variable number of grid elements with one or two consumers each (see Figure 1).

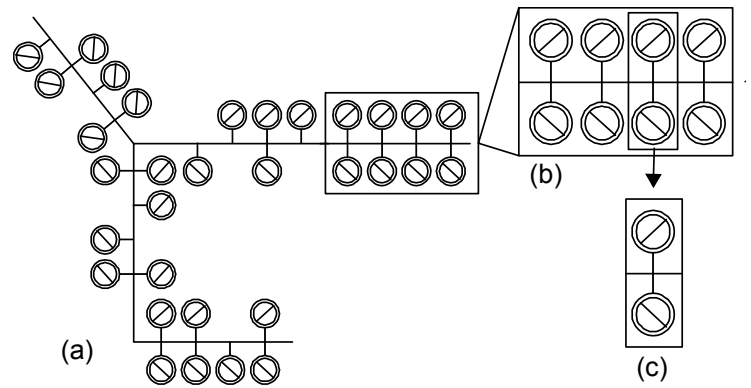

Figure 1: Demand structure (a - distribution grid, $\mathrm{b}$ - grid segment, $\mathrm{c}$ - grid element)

In the presented modelling approach, one GC represents one grid segment. The GC enables the modelling of a grid segment by creating a series of grid elements using arrays of models. For the GC, the used technologies, number of consumers as well as grid structure parameters such as length or type of cables are freely exchangeable. By connecting several GC, each representing a grid segment, a distribution grid can be modelled. Each of the GC used in the modelling of the distribution grid can be individually configured regarding technology as well as properties for the grid structure like cable type or length. Due to the interchangeability of parameters and technologies inside the GC, case studies of changing configurations of grid structures and used technologies of existing energy grids as well as future scenarios are possible.

Each GC consists of an array of grid element models (GridElement (GE)). Depending on user input, one or multiple GE are strung together to create a grid segment. The basic idea of the GE model is that each grid element ultimately consists of an energy demand (electricity, space heating and hot water) and technologies to meet that demand and/or to generate additional energy (see Figure 2). Each of the consumers inside the GE is represented by its own energy demand and technologies. For each GE, either one or two consumers can be activated. In this way, an exact representation of the demand and grid structure is possible as well as a simplified representation.

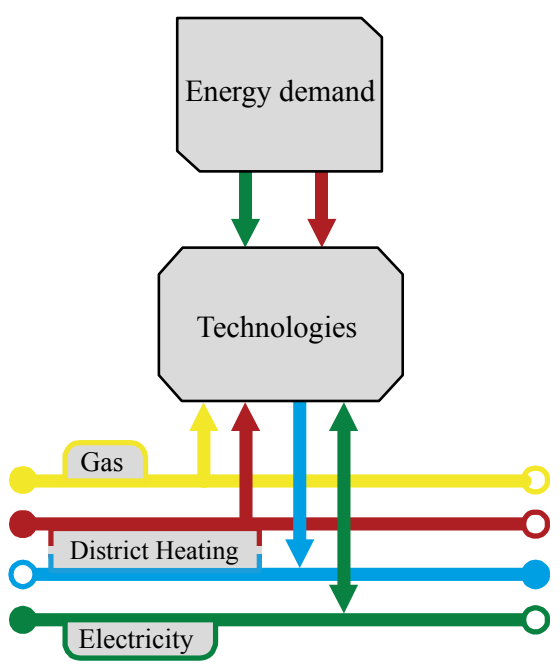

Figure 2: Basic idea behind the GridElement structure and connection scheme for one consumer inside a GridElement.

The GE model consists of sub-models providing the mentioned demand, representing used technologies and grid structures inside the grid element (see Figure 3). This includes:

- Gas and district heating pipe models

- Electric cable models

- Models representing used technologies

- Models providing time series of energy demand

Simulations of gas distribution networks are enabled by the use of gas pipe models taken from the Modelica library TransiEnt and adjusted to calculate the pressure drop not from nominal values, but via the DarcyWeißbach equation with the function StraightPipe.dp_overall_MFLOW provided by the FluidDissipation library inside Modelica. The gas pipe is parametrised regarding length and diameter. Changing composition of the gas, for example through hydrogen injection, is accounted for.

The electric grid is modelled by using an electric cable model from TransiEnt library. The type of cable (diameter, material, resistance) is freely exchangeable via use of replaceable models.

For simulations of district heating networks, three models representing district heating pipe pairs are placed inside the GE as replaceables. The central pipe model represents the distribution pipe of the heat carrier 
in the grid element. For the connection of consumers to the distribution pipe, two pipe models are placed perpendicular to the distribution pipe. If faster simulations are desired or physical effects in the house pipes are neglected, the models of the house connections may be deactivated. The use of parallel pipe models increases usability by parameterising the pipes homogeneously for length and geometry. Alternatively, the user is able to specify the nominal diameter based upon manufacturer specifications saved within records inside Modelica from which the pipe geometry is set. Initialization parameters for temperature and pressure for supply and return pipes are provided on the top level of the modelled energy grid.

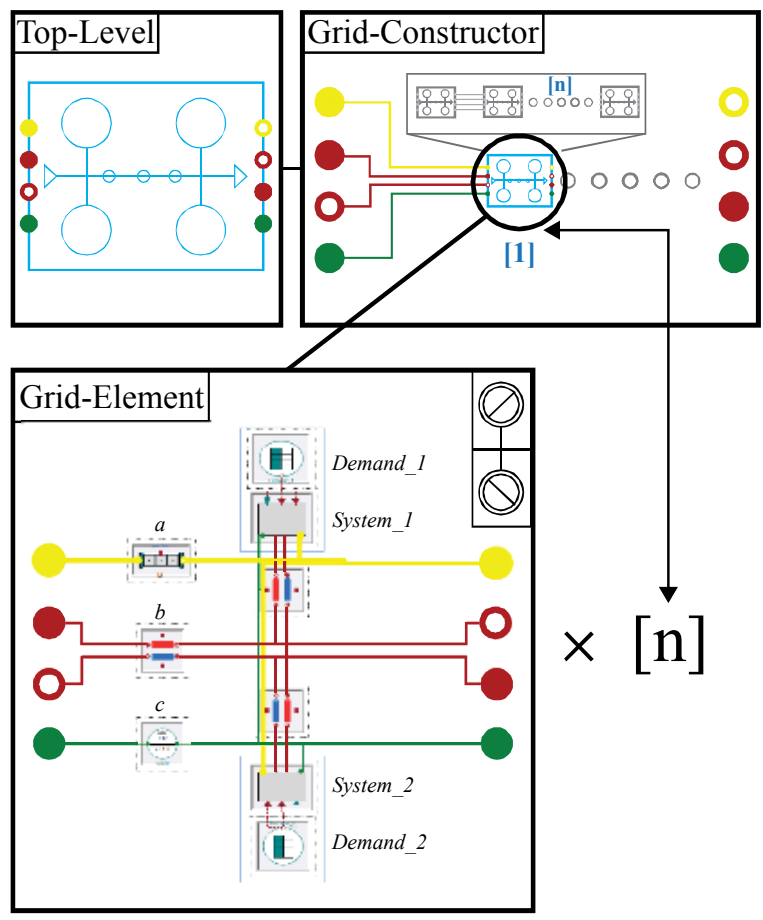

Figure 3: Structure of the GridConstructor and GridElement. a - gas pipe model, $\mathrm{b}$ - district heating pipe model, c - electric cable model.

The district heating pipes used inside the GE are modelled after the PlugFlow approach described in (Heijde, 2017; Hägg, 2016). Contrary to pipe models with spatial discretisation, the PlugFlow approach determines the fluid properties only at the inlet and outlet of the pipe by means of the residence time. Temperature wave propagation and heat loss are both handled by use of the spatialDistribution() function included in Modelica. Validation work carried out for the PlugFlow approach in (Heijde, 2017) and as part of simulations within the IntegraNet shows good accuracy to measurements.

Pressure drop calculations inside the pipe model are carried out with the Darcy-Weißbach equation using the function StraightPipe.dp overall MFLOW provided by the FluidDissipation library. Fluid properties such as density or specific heat capacity are calculated with models from the TILMedia suite developed by the TLKThermo GmbH.

Electric cables, gas pipes and district heating pipes can be deactivated or activated as required. Corresponding connectors are deactivated automatically. Multiple GE inside a GC and sub-models inside of a GE are connected automatically.

The technologies used inside the GE are integrated in models called Systems_1 and Systems_2 representing technologies used for the top consumer $(1)$ and bottom consumer (2) of the grid element. Consumers inside a grid segment are divided into a top row and bottom row representing the two sides of a road. Inside each of the System models multiple models for technologies like gas boilers, photovoltaics or heat pumps are activated or deactivated based upon the settings defined during parametrisation of the GC.

For the energy demand, time series of heat and/or cooling and electricity demand with arbitrary resolution (s, min, h) are used and provided by the models Demand_l and Demand_2 for both consumers. These time series are saved inside comma separated value (csv) files and may be provided for the model outside Modelica based upon real life measurements, computer generated data or standard load profiles. Technology models defined inside System use these profiles to meet the associated demand.

\subsection{Programming \& Modelling details}

To enable flexible modelling of grid segments, the necessary programming effort can be divided into the following problem statements:

- Enabling the creation of the demand and grid structure

- Exchangeability of technologies

- Enabling a user-friendly parametrisation

The demand and grid structure inside the grid segment are achieved by use of Boolean arrays. For this, the user has to define the number of grid elements $n$ inside the grid segment. From this, $n$ GE models are initialized inside a GC model (see Figure 4).

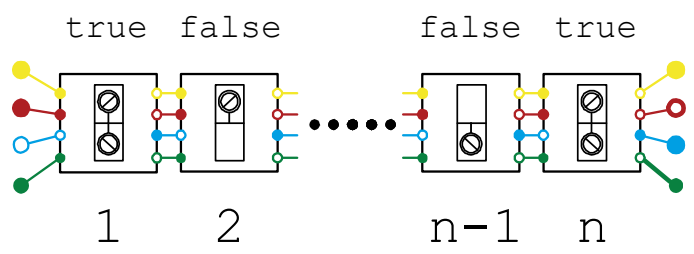

Figure 4: Arrangement of GE models inside a GC

By use of for-loops, automatic connections of GE inside the $\mathrm{GC}$ are achieved:

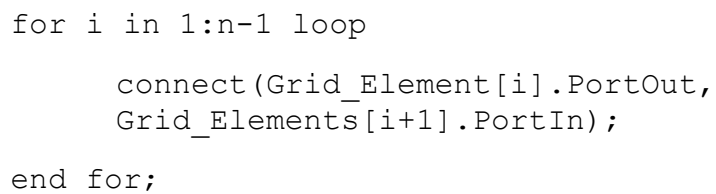


The inlet connectors for the first GE $(\mathrm{n}=1)$ and the outlet connectors for the last GE (n) are connected to the connectors of the GC by regular connect statements. All connectors and their interconnections are dependent on booleans, which are set depending on the technologies used. Also, the models for gas pipes, district heating pipes and cables are conditional models and are activated/deactivated depending on parametrisation. Therefore, unused models are removed from the equations during compilation.

In each GE, one or two consumers can be connected to the energy grid. To achieve this property, an array of Booleans second_Consumer[:] is created with which all models belonging to the second consumer are activated if the boolean is true (see Figure 4). This Boolean is always set false if only one consumer is supposed to exist in the related GE, no matter if it's the top or bottom consumer.

By use of records, properties of the GE models inside the GC are set. The records are defined to have all the properties associated to a given type of parametrisation objective. For example, length and cable type of the electric cable models inside each GE are set as array parameters inside the record CableParameters. The length of the cable model in each GE inside a GC is assigned as follows:

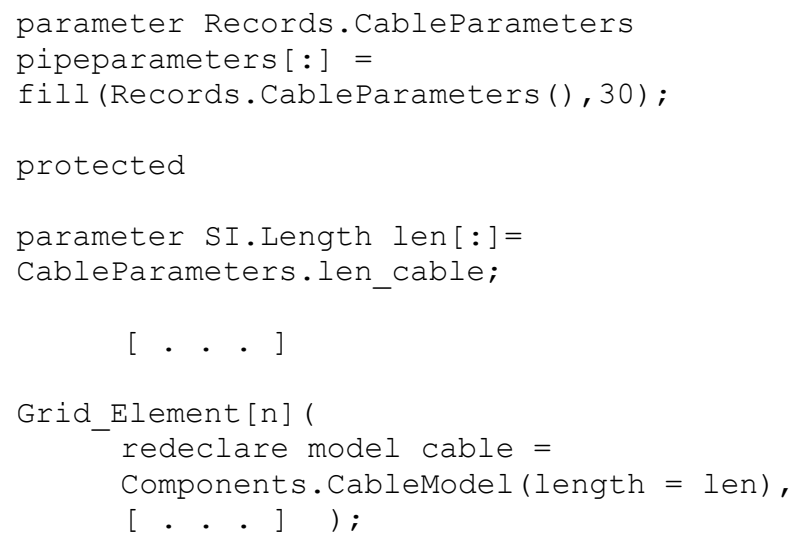

Records are not only used for the parametrisation of models representing grid structure, but also for the exchangeability of technologies in the models System_1 and System_2. A record called TechnologyMatrix with parameters as integers for all available technologies is created. This record is used for each consumer (top and bottom) as a matrix with columns representing technologies and rows representing each GE. The set parameters inside this matrix are propagated from the GC to the corresponding GE and corresponding technology. With these propagated integers (zero or one), technology models like gas boilers or photovoltaic are activated or deactivated with if-Statements. Integers are used instead of booleans to reduce the time the user needs to enter the parameters. Technologies get activated with one and deactivated with zero. For this approach to work, all possible technology models must be present as default in the System model, but each of the models can be deactivated freely. Connectors inside the model System ( 1 and 2) are activated and deactivated based upon used technologies without user-input. For example, district heating connectors are only activated if technologies corresponding to district heating are used. The values inside the TechnologyMatrix can be freely changed from simulation to simulation.

Parametrisation of technologies in System_l and System_ 2 for properties like efficiency is realised by use of further records containing the parameters used in the individual models. Set parameters are propagated to activated technologies and used as input for the models with the redeclare model statement.

The demand time series data is provided to System with a model CombiTimeTable each inside the model Demand 1 and Demand 2. In order for each of the consumers to use their own energy demand as a time series, the data for space heating, domestic hot water heating and electricity must be stored inside three csv tables with each column representing another consumer. Alternatively, the demand time series can be provided in a single csv-file, which contains groups of columns with each column group specifying the demand data for one consumer. Each group consists of three columns that specify the data for the electricity, space heating and domestic hot water demand respectively. The time series for the energy demands are assigned to the Demand models for each GE by accessing the corresponding column in the provided time table. Columns are accessed and assigned in series starting from a user defined integer start_cl and start_c2 representing the top and bottom consumer:

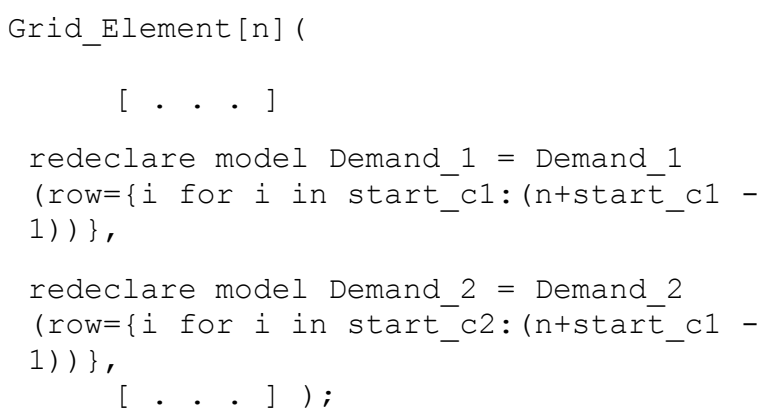

\subsection{Parametrisation}

The parametrisation of one GC can be divided into the following steps:

1. Define number of grid elements $(n)$ as well as the arrangement of consumers (secondConsumer)

2. Deactivate/activate inlet and outlet connectors

3. Set properties of energy distribution models

4. Assign load-profiles

5. Define and set properties of technologies used

Parametrisation of the GC starts with the definition of grid elements in the given grid segment. As an example, Figure 5 shows a grid segment consisting of six grid 
elements with one or two consumers per grid element. Based on this example, the user passes $n=6$ to the $\mathrm{GC}$ and arranges the consumers on the grid element by setting the booleans for secondConsumer. The booleans secondConsumer are stored in an array with the alignment orientation source to sink. The orientation of the consumer to the street for secondConsumer is irrelevant and does not influence the simulation (see Figure 5).

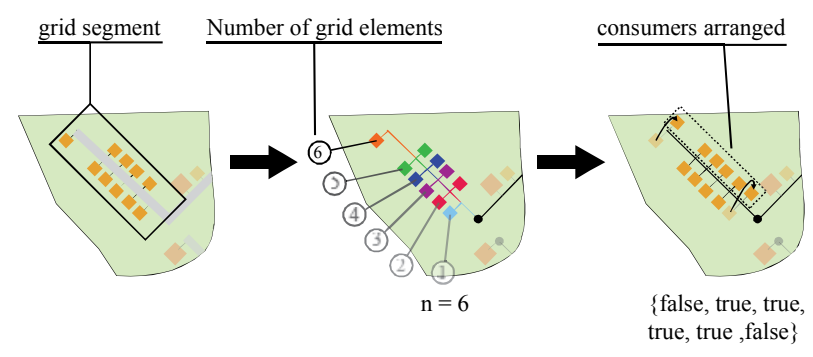

Figure 5: Graphical representation of the first steps for parameterizing the GC

As a next step, the user deactivates unused connectors of the $\mathrm{GC}$ with checkboxes. For example, the gas inlet and outlet connectors are not necessary if a district heating network and electricity network without gas network is to be simulated. As mentioned, this also automatically deactivates any connector and energy distribution model associated to the deactivated connectors. If previous GC models have already been placed and parameterised, the GC can be connected to the corresponding $\mathrm{GC}$ via the respective connectors.

Subsequently the properties of energy distribution models (gas pipes, cables and district heating pipes) are set by editing the corresponding records. Each row inside the array of records represents one of the grid elements (see Figure 6).

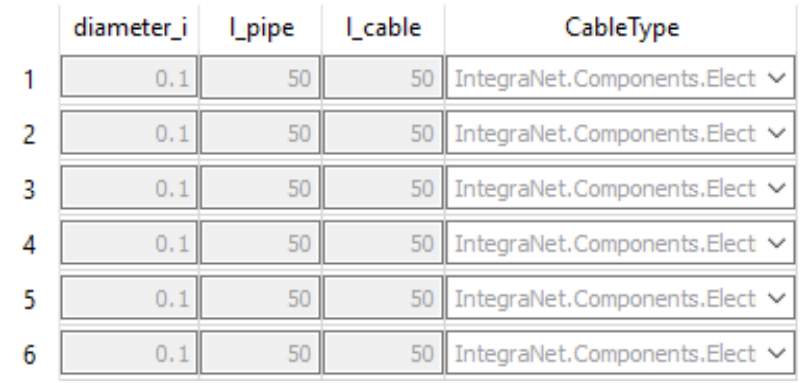

Figure 6: Parametrisation of gas pipes and electric cables models inside a GC

With the basic structure of the grid segment set, the user is able to pass the load profiles for heat and electricity demand to the GC. For this, the path to the csv-tables is set for the top consumers and bottom consumers. Consumers virtually transformed to exist in the top row during the arrangement of secondConsumer have to have their corresponding load profile inside the csv-table for the top row even though they, in reality, are placed on the other side. If the demand profile tables are used in more than one GC, the start column of the load profiles has to be passed as the integer start_cl and start_c2.

Finally, the technologies used in each network element of the GC are defined by using two arrays of the record TechnologyMatrix for the upper and lower rows of consumers. Technologies for the consumers are deactivated/activated with ones (activated) and zeros (deactivated). Analogue to the parametrisation of the pipes and cables, each row inside the parameter array represents a grid element and consumer (see Figure 7). Parameters of the activated technologies are set with further records similar to the parameterisation of gas pipe, cable and district heating pipe models.

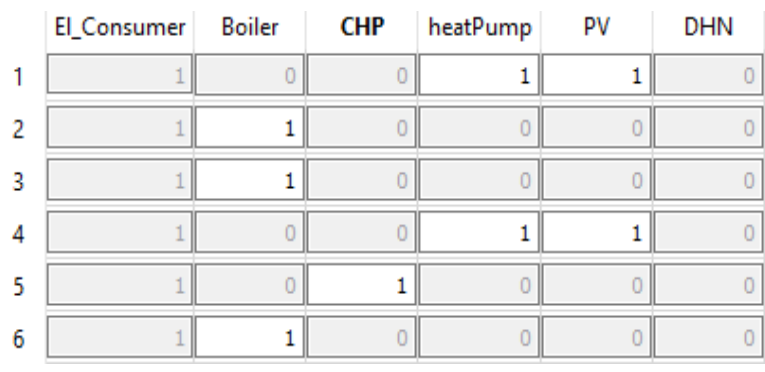

Figure 7: Defining technologies used in the GC for each GE with use of a record

With the GC fully parametrised and connected to other corresponding GC models, the simulation of the desired energy grid is possible.

Currently, the parametrisation is the most timeconsuming part of modelling an energy grid, which is considerably simplified by the GC approach.

\section{Use Cases}

In the following section, utilizations of the $\mathrm{GC}$ for simulation of energy grids are presented. At first, a coupled gas and electric grid simulation of a rural district is described. Subsequently, the simulation of a low temperature district heating network (DHN) is described with the aim of investigating heat losses and potentials for Power-to-Heat (PtH).

\subsection{Simulation of a coupled gas and electricity grid}

In the following the first use case and the general functionality and usability of the provided models are presented. General challenges of modelling a coupled electric power and gas distribution grid with different technologies such as photovoltaic (PV) or Power-to-Gas $(\mathrm{PtG})$ are shown.

In many research projects, no real measurement data of the demands per building is available. To anyhow introduce high fluctuations into the modelled district, profiles for the heat demand according to the VDI 4655 (Verein Deutscher Ingenieure, 2008) can be used. Using the same profile for each building would lead to unrealistically high gradients and maxima of the total 
demand. Thus, to also depict needs to balance between the consumers, it is necessary to shift the VDI-profiles individually for each building over time. For shifting a normal distribution is used.

One challenge when simulating large networks, is to secure stable initialisation. Especially when using parallel and serial networks of gas pipes, the initialisation of the pressure loss for the given heat demands at the first-time step is fairly difficult. To avoid having to calculate the complete network in a static cycle beforehand, initialisation using zero heat demand, resulting in zero gas flow, can be used. This increases the usability of the model, especially when changing the grid structure frequently due to different scenarios.

The described modelling approach has successfully been used to simulate and analyse a rural example district containing 314 buildings - all of them connected to the electric grid and 166 buildings also supplied with gas.

Figure 8 shows the resulting electricity surplus of this grid with PV for one day each of different seasons of the year.

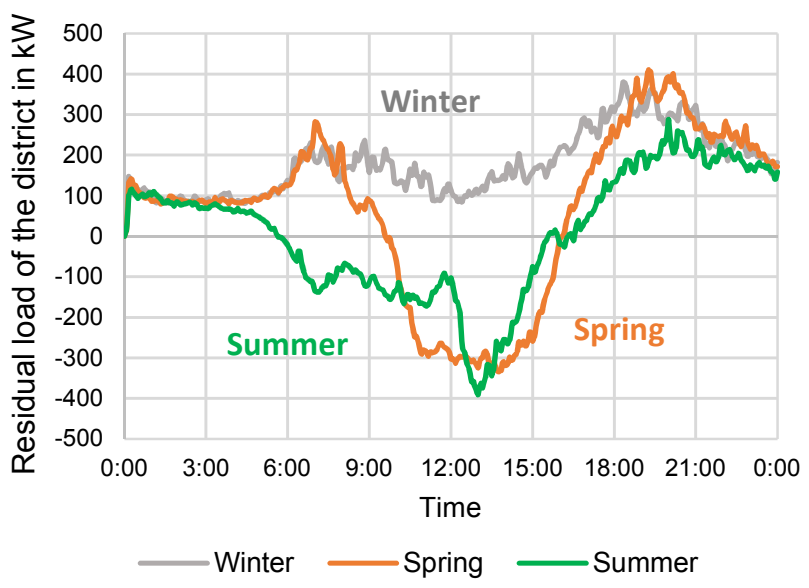

Figure 8: Total electricity load for the simulated example district with PV for three different days of the year

The simultaneous simulation of the coupled electric power and gas grid enables the analysis of sector coupling technologies, such as PtG. Converting all surplus electric power into hydrogen and feeding it into the gas grid leads for the observed grid for the spring day to the curves as depicted in Figure 9. The resulting volume fraction of hydrogen of up to $64 \%$ exceeds the admissible range clearly. Implementing a monitoring of the gas properties and corresponding control strategies, enables the analysis of the resulting residual load with different limit values for the gas properties.

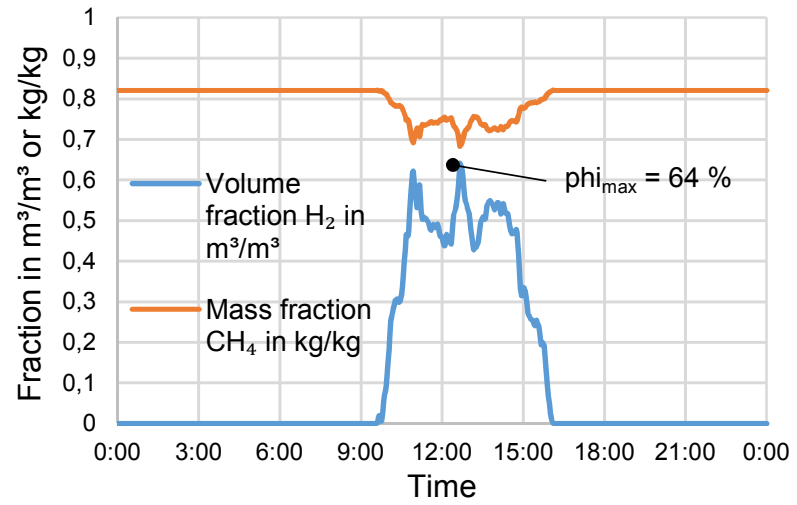

Figure 9: Hydrogen feed-in into the gas grid from $\mathrm{PtG}$ without control of the gas properties at a spring day

Using the same basic grid, different scenarios with various combinations of technologies (conventional heating systems, PV, CHP-systems and PtG) have been simulated including variations of the corresponding automation and control strategies.

Thus, the presented modelling approach enables a straightforward comparison of the different supply and control strategies.

Further details on the modelling of this specific grid and simulation results can be found in (Garzon-Real et $a l, 2018)$.

\subsection{Simulation of a district heating network}

Besides the coupled electrical and gas grid, the GC was used to simulate and examine a planned low temperature DHN regarding heat losses and potentials for PtH. The examined DHN is based upon the use of a heat source providing heat to achieve a supply temperature of around $25^{\circ} \mathrm{C}$ all year round. This supply temperature is elevated to the necessary temperature levels for domestic hot water and room floor heating by use of heat pumps at high coefficients of power. Pipes planned for the network are regular uninsulated polyethylene pipes embedded in a filling of sand. The DHN is supposed to provide heat to a district with around 200 consumers made up of residential and commercial energy-efficient buildings.

Modelling of the heating network was carried out according to the specifications of the network and demand structure given by the project. PtH was considered in the combination of heat pumps and PV systems. For this, two scenarios of the DHN with 100 and $50 \%$ utilization of the available roof areas for PV are investigated. Standard load profiles are used for the heat demand and a combination of measurement data and computer-generated load profiles for the electricity demand. Real-life weather data for solar radiation as well as ground and air temperature provided by the German weather service (Deutscher Wetterdienst, 2017) were taken from weather stations closest to the planned area of the DHN. 
The DHN has been simulated for one year with an hourly resolution, resulting in computing times of around $6 \mathrm{~h}$ for a single simulation and $13 \mathrm{~h}$ for three parallel simulations of different scenarios. Deactivation of house pipes can reduce the simulation run time further.

Simulation of the heating network resulted in a heat demand of $6700 \mathrm{MWh} / \mathrm{a}$ with a peak load of $2500 \mathrm{~kW}$ (see Figure 10). The heat loss of the DHN was determined to be $25 \%$ of the annual heat supplied to the DHN (1675 MWh/a). Analysis of the DHN simulation revealed low heat carrier velocities due to oversized pipes and thus increased heat losses.

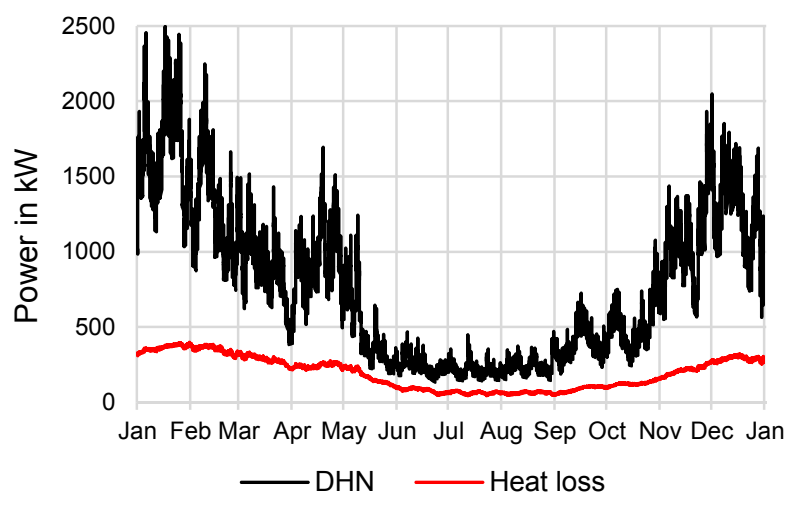

Figure 10: Simulated heat load of the DHN and corresponding heat loss profile

Complementary, the results of the simulation made it possible to determine the transition period from winter to summer as the critical time for operation of the DHN. Due to sudden increases in outside temperature during this transitional period, the heat demand of the DHN drops significantly. With lowered heat demand the mass flow through the DHN is decreased resulting in a significant cooling of the heat carrier inside the pipes. In this context, periods of high temperatures during the transition period, followed by sudden drops in temperature can lead to a DHN operation where it is not possible to immediately provide heat to the consumer until the heat carrier temperature is increased again.

As mentioned, the DHN is simulated coupled with the electrical grid to examine the potentials for PtH. The electricity demand of the district is $6600 \mathrm{MWh} / \mathrm{a}$. At $100 \%$ utilization of the available roof areas with PV, 27 $\%$ of the electricity demand is met. For the $50 \%$ scenario, $13 \%$ of the electricity demand is met by the PV systems. Due to the contradictory nature of heat demand and electricity generation of PV systems, the potential of heat pumps to lower the negative residual load is low (see Figure 11).

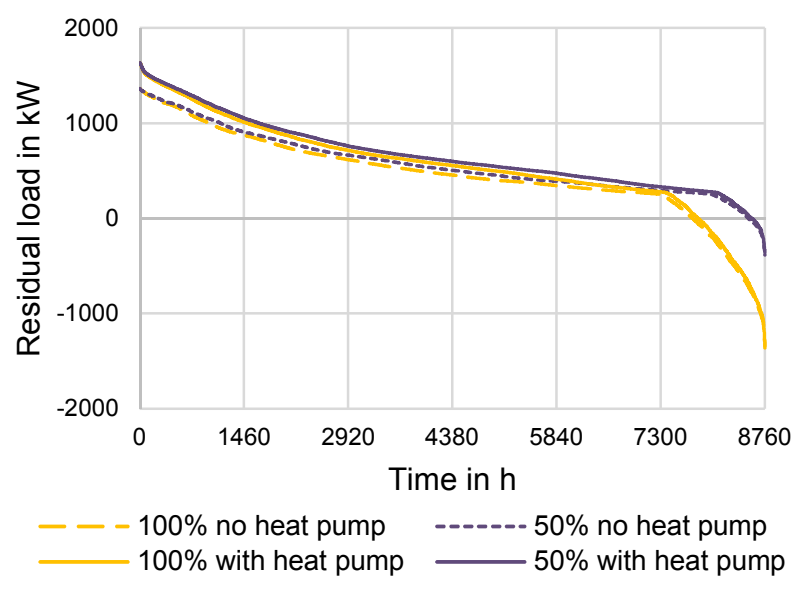

Figure 11: Sorted residual load for both roof utilization scenarios with and without heat pump electricity demand

\section{Summary and Outlook}

The approach of demand oriented modelling of urban frameworks and the corresponding energy grids was presented. The general modelling philosophy was explained and described in its realisation and the fundamental development ideas were shown in detail. In conclusion first examples are presented and can give a first view on the potentials of the work.

In the future several additional features will be realised.

For example, advances during the development regarding the design of the $\mathrm{GC}$ make it possible to further reduce the time needed for parametrisation. By use of identification integers (ID) the import of parametrisation data from Geographical Information Systems (GIS) saved inside Excel files is going to be automated. Each consumer in the desired energy grid gets assigned an ID from which data used for parametrisation as well as corresponding load profiles are imported into the GC models in Modelica. Based on this approach, the automatic generation of GC models with the help of Excel is currently being developed. Additionally, a direct import of GIS data into Modelica is conceptualised using the programming language Python.

All shown work will be published within a future release of the TransiEnt library.

\section{Acknowledgements}

The authors thank the German Federal Ministry for Economic Affairs and Energy for funding the project »Integrierte Betrachtung von Strom-, Gas- und Wärmesystemen zur modellbasierten Optimierung des 
Energieausgleichs- und Transportbedarfs innerhalb der deutschen Energienetze - IntegraNet« (FKZ 0324027B).

\section{References}

L. Andresen, P. Dubucq, R. Peniche, G. Ackermann, A. Kather, G. Schmitz. Abschlussbericht des Verbundvorhabens: Transientes Verhalten gekoppelter Energienetze mit hohem Anteil Erneuerbarer Energien. Technische Informationsbibliothek, Hannover. 2017. doi: 10.2314/GBV:1002659345

M. Behnert, A. Hartke, T. Bruckner Spannungsfeld Netzstabilität - Lehren aus vergangenen Blackouts für eine sichere zukünftige Stromversorgung. Energiewirtschaftliche Tagesfragen, No. 9, pp. 10-14, 2018.

M. Blesl. Räumlich hoch aufgelöste Modellierung leitungsgebundener Energieversorgungssysteme zur Deckung des Niedertemperaturwärmebedarfs. Universität Stuttgart-IER, 2002.

Deutscher Wetterdienst (DWD). Measurements from Climate Data Center (CDC). 2017.

J. Garzon-Real, B. Dahlmann, M. Zdrallek, J. Hüttenrauch, M. Wupperfeld, J. Benthin, A. Heyer, F. Burmeister, R. Albus, W. Köppel, K. Peters. Entwicklung und Validierung eines kombinierten Strom- und Gasnetzautomatisierungskonzepts auf Verteilnetzebene. gwf Gas + Energie, No 10, pp.68-81, 2018.

B. Heijde, M. Fuchs, et al. Dynamic equation-based thermohydraulic pipe model for district heating and cooling systems. Energy Conversion and Management, No. 151, pp. 158 - 169, 2017. doi: 10.1016/j.enconman.2017.08.072.

R. Hägg. Dynamic Simulation of District Heating Networks in Dymola. Masterthesis, Department of Energy Sciences, Lund Universitet, 2017.

IntegraNet, https://www.integranet.energy/, Fraunhofer UMSICHT, Gas- und Wärme-Institut Essen e.V., 2018.

TransiEnt, https://www.tuhh.de/transient-ee/, Technische Universität Hamburg Harburg, 2018.

Verein Deutscher Ingenieure. VDI 4655 Referenzlastprofile von Ein- und Mehrfamilienhäusern für den Einsatz von KWK-Anlagen. Beuth Verlag GmbH, 2008. 